International Journal of Pure and Applied Mathematics

Volume 111 No. 1 2016, 17-29

ISSN: 1311-8080 (printed version); ISSN: 1314-3395 (on-line version)

url: http://www.ijpam.eu

doi: 10.12732/ijpam.v111i1.3

\title{
SQUEEZE FILM CHARACTERISTICS AND STEADY STATE PERFORMANCE OF A PARABOLIC INCLINED POROUS SLIDER BEARINGS USING COUPLE STRESS FLUID
}

\author{
Nisha $^{1 \S}$, Sundarammal Kesavan ${ }^{2}$, S. Sangeetha ${ }^{3}$ \\ ${ }^{1,2,3}$ Department of Mathematics \\ Faculty of Science \& Humanities \\ SRM University \\ Kattankulathur, 603 203, Tamil Nadu, INDIA
}

\begin{abstract}
On the basis of the micro continuum theory, a theoretical analysis of the effects of pressure distribution using couple stress on the squeeze film behavior of parabolic inclined slider bearing is presented. The modified Reynolds equation governing the squeeze film pressure is derived by using the stokes constitutive equation and the analysis taking into account the squeezing effect with no slip at the porous interface comparing with the traditional Newtonian lubricant case, the effects of couple stress characteristics by the couple stress parameter signify an improvement in the steady state performance. We are considering the parabolic inclined bearing to analyze the effect of pressure and compared to the inclined plane bearing, step inclined bearing, the parabolic shaped slider bearing lubricated with couple stress fluids results in a higher pressure distribution.
\end{abstract}

The performances are emphasized especially for the bearing with smaller film heights.

AMS Subject Classification: 76B99, 76M15, 76M25, 76N99, 76S05

Key Words: couple stress fluids, parabolic shaped film bearing, micro-continuum theory, squeeze velocity

Received:

Revised:

September 23, 2016

Published: $\quad$ November 30, 2016

$\S$ Correspondence author (c) 2016 Academic Publications, Ltd. url: www.acadpubl.eu 


\section{Introduction}

Slider bearings are often designed for supporting the transverse load in engineering applications. The shape of the slider bearing is one of the major geometric conditions influencing the performance of the bearing. Since the conventional continuum theory can't describe the nature of flow accurately, the micro-continuum theories and stokes theory [12] came into account in which the presence of couple stress, body couples takes into account.

Thus, the micro-continuum mechanism of fluids is generalized by the polar theory of complex fluids, which is characterized by classical Cauchy stress as well as couple stress in fluids.

The squeezing film technology is widely observed in applications of engineering practices such as human joints, dampers, matching gears etc.

From the studies of squeezing effects given by J.R. Lin, R.F. Lu (2003) taking into account the transient squeezing-action effect and considering the couple stress effects resulting from the lubricated blended with various additives, the non Newtonian dynamic Reynolds equation has been derived by applying the stokes micro-continuum theory. Another study by P.S. Rao, S. Aggarwal (2014) establishes that from the bearing life period point of view the surface roughness must be accounts for while designing the bearing system even if a suitable value of couple stress parameter has been chosen.

Many researchers considered parabolic shaped slider bearing, in 2004, J.R. Lin, Y.M. Lu, concluded that compared with the inclined plane bearing case, the parabolic shaped slider lubricated with couple stress results in a higher load carrying capacity and a smaller required volume flow rate especially for the bearing designed at a larger value of the profile parameter. In slider bearings in Figure (a), the surface has related tangential velocity, the upper plate carries a load and the lower surface moves relative to the upper one. The relative motion of the surfaces induces velocity gradient in the fluid film. This velocity gradient give rise to viscous force in the fluid film. To match this frictional force, pressure gradient is developed resulting in the load capacity of the bearing.

The squeeze film behavior arises from the phenomenon of two lubricated surfaces approaching each other with a normal velocity. Because the viscous lubricant contained between these two surfaces can't be instaneously squeezed out, it takes a certain time for these surfaces to come into contact. A typical application of squeeze film technology is a squeeze film vibration absorber in an aircraft jet engine. Here the engine shaft rotates at high speed in ball bearing races. 


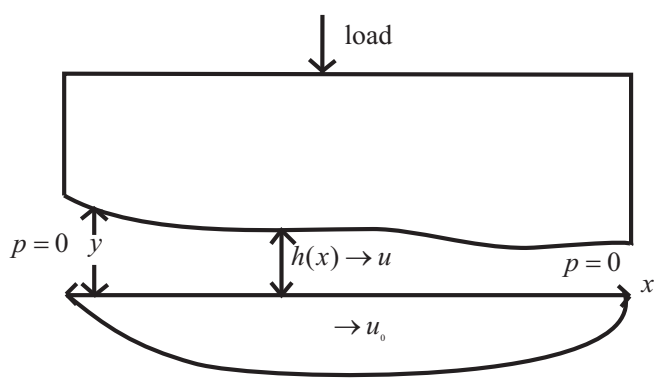

Figure (a)

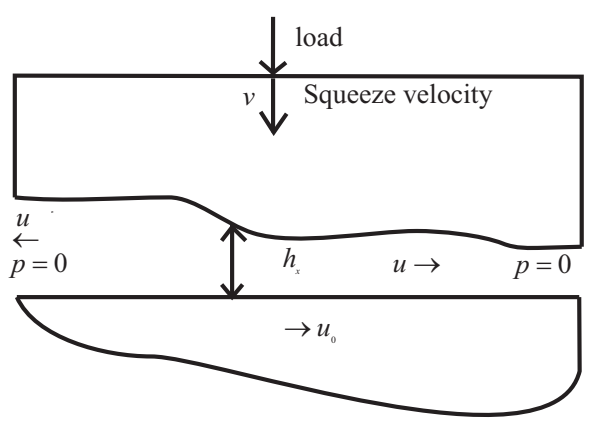

Figure (b)

\section{Mathematical Form}

The modified Reynolds equation type equation for the pressure in the film region is obtained by using equation (3) for $u$ in the continuity equation and then integrating over the film thickness using the boundary condition:

\section{Analysis}

The slider bearing of length $\mathrm{L}$ with velocity $\mathrm{U}$ is considered in Figure (c) and the parabolic film profile is described by:

$$
h(x)=h_{m}+h_{p}(x),
$$




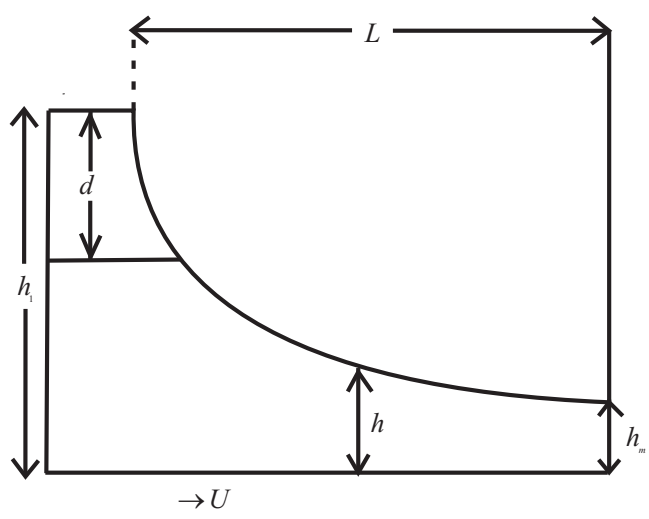

Figure (c)

where $h_{m}$ is the minimum film thickness at the outlet and denotes the shoulder height of the bearing and

$$
h_{p}=\frac{d}{L}\left(L-2 x+\frac{x^{2}}{L}\right) .
$$

Under the assumptions that the lubricant film is thin, the body forces and body couples are absent, the continuity equation and motion equation can be expressed in a non-dimensional form:

$$
\left.\begin{array}{l}
\frac{\partial p}{\partial x}=\mu \frac{\partial^{2} u}{\partial y^{2}}-\eta \frac{\partial^{4} u}{\partial y^{4}}, \\
\frac{\partial p}{\partial y}=0, \\
\frac{\partial u}{\partial x}+\frac{\partial v}{\partial y}=0 .
\end{array}\right\}
$$

Here the non dimensional quantities are defined by:

$$
x^{*}=\frac{x}{l}, \quad z^{*}=\frac{z}{h_{0}}, \quad u^{*}=\frac{u}{U}, \quad p^{*}=\frac{p h_{0}^{2}}{\mu U L}, \quad l^{*}=\frac{l}{h_{0}} .
$$

where $u^{*}$ represent dimensional velocity component in the $x$ direction, $p^{*}$ is the pressure, $\mu$ is the shear velocity, and $\eta$ is a new material constant and it is responsible for the couple stress property.

If $\eta=0$, which makes $l^{*}=0$ the classical form of a Newtonian lubricant which is from $(3)$. 
At the fluid porous interface

$$
y=0, \quad u=U+\frac{1}{s} \frac{\partial u}{\partial y}, \quad v=-v_{1}, \quad \frac{\partial^{2} u}{\partial y^{2}}=0 .
$$

At the upper solid surface

$$
y=h, \quad u=0, \quad v=\frac{d h}{d t}, \quad \frac{\partial^{2} u}{\partial y^{2}}=0 .
$$

The solution of (3) subject to boundary conditions (4), (5) is given by

$$
\begin{aligned}
u^{*}=\frac{1}{2 \mu} \frac{\partial p^{*}}{\partial x^{*}}\left\{\left(y^{*}-h^{*}\right)[\right. & \left(y+h^{*} \xi-2 l^{*} \tanh \left(\frac{h^{*}}{2 l^{*}}\right)\right. \\
& \left.\left.\left.+2 l^{* 2}\left[1-\cosh \left(\frac{\frac{2 y-h^{*}}{2 l^{*}}}{\frac{h^{*}}{2 l^{*}}}\right)\right]\right)-U s\left(y-h^{*}\right) \xi\right]\right\},
\end{aligned}
$$

Where $l$ is called couple stress parameter and is equal to $\sqrt{\frac{\eta}{\mu}}$ and $\xi=\frac{1}{1+h s}$.

Using Leibnitz rule we get

$$
\frac{\partial}{\partial x^{*}}\left[f^{*}\left(h^{*}, l^{*}\right) \frac{\partial p^{*}}{\partial x^{*}}\right]=12 \mu\left(\frac{-k}{1-\beta}\right)+6 \mu U \frac{\partial h^{*}}{\partial x^{*}}
$$

where

$$
\begin{aligned}
f^{*}\left(h^{*}, l^{*}, \xi\right)=h^{* 3}(1+3 \xi)-6 h^{* 2} l^{*} \xi \tanh & \left(\frac{h^{*}}{2 l^{*}}\right) \\
& -12 l^{* 2} h^{*}+24 l^{* 3} \tanh \left(\frac{h^{*}}{2 l^{*}}\right) .
\end{aligned}
$$

As the value of $l$ approaches zero, the function $f$ yields the result of $h^{3}$, the lubrication equation reduces to the classical form for a Newtonian $N=0, l=0$ lubricant.

Couple stress flow is governed by the Darcy law given by Naduvinamani et al.

$$
\begin{aligned}
& u_{1}=\frac{-k}{\mu(1-\beta)} \frac{\partial p^{*}}{\partial x^{*}}, \\
& v_{1}=\frac{-k}{\mu(1-\beta)} \frac{\partial p^{*}}{\partial y^{*}},
\end{aligned}
$$


where $u_{1}, v_{1}$ are modified Darcy velocity components along $x, y$ directions on the porous region where $p$ is the pressure. Integrating the above equation with respect to $x$ yields,

$$
\begin{aligned}
& p=\int_{x^{*}=0}^{x^{*}} \frac{6 \mu U h^{*}}{h^{* 3} f\left(h^{*}, l^{*}, \xi\right)}-12 \int_{x^{*}=0}^{x^{*}} \frac{\mu v_{1} x^{*}}{h^{* 3} f\left(h^{*}, l^{*}, \xi\right)} \\
& +c_{1} \int_{x^{*}=0}^{x^{*}} \frac{1}{h^{* 3} f\left(h^{*}, l^{*}, \xi\right)}, \\
& f_{1}=\int_{x^{*}=0}^{x^{*}} \frac{\mu U h^{*}}{h^{* 3} f\left(h^{*}, l^{*}, \xi\right)}, \\
& f_{2}=\int_{x^{*}=0}^{x^{*}} \frac{\mu v_{1} x^{*}}{h * 3 f\left(h^{*}, l^{*}, \xi\right)}, \\
& f_{3}=\int_{x^{*}=0}^{x^{*}} \frac{1}{h * 3 f\left(h^{*}, l^{*}, \xi\right)} .
\end{aligned}
$$

Therefore $p$ takes the form

$$
p=6 f_{1}\left(x^{*}, h_{m}^{*}\right)-12 f_{2}\left(x^{*}, h_{m}^{*}\right)+c_{1} f_{3}\left(x^{*}, h_{m}^{*}\right) .
$$

The integral constant $c_{1}$ can be evaluated by using the pressure $=0$ condition at the outlet.

$$
c_{1}=12\left(\frac{f_{2}\left(x^{*}, h_{m}^{*}\right)}{f_{3}\left(x^{*}, h_{m}^{*}\right)}\right)-6\left(\frac{f_{1}\left(x^{*}, h_{m}^{*}\right)}{f_{3}\left(x^{*}, h_{m}^{*}\right)}\right) .
$$

Evaluating the lubrication film thickness at the location where the pressure gradient is equal to zero, gives (12) the following position.

The non dimensional load carrying capacity is computed by integrating the film pressure over the film region

$$
W^{*}=\frac{W h_{0}^{2}}{\mu U L^{2} B}=\int_{x^{*}=0}^{1} P^{*} d x^{*},
$$

where $B$ denotes the width of the bearing.

On performing the integration results in

$$
W^{*}=6 F_{\Lambda}\left(h_{m}^{*}\right)-12 F_{B}\left(h_{m}^{*}\right)+C_{1} F_{C}\left(h_{m}^{*}\right),
$$

where

$$
F_{\Lambda}\left(h_{m}^{*}\right)=\int_{x^{*}=0}^{1} f_{1} d x^{*}
$$




$$
\begin{aligned}
& =\int_{x^{*}=0}^{1} \int_{x^{*}=0}^{x^{*}} \frac{\mu U h^{*}}{h^{*^{3}} f\left(h^{*}, l^{*}, \xi\right)} d x^{*} d x^{*} \\
F_{B}\left(h_{m}^{*}\right) & =\int_{x^{*}=0}^{1} f_{2} d x^{*} \\
& =\int_{x^{*}=0}^{1} \int_{x^{*}=0}^{x^{*}} \frac{\mu v_{1} h^{*}}{h^{*^{3}} f\left(h^{*}, l^{*}, \xi\right)} d x^{*} d x^{*} \\
F_{C}\left(h_{m}^{*}\right) & =\int_{x^{*}=0}^{1} f_{3} d x^{*} \\
& =\int_{x^{*}=0}^{1} \int_{x^{*}=0}^{x^{*}} \frac{1}{h^{*^{3}} f\left(h^{*}, l^{*}, \xi\right)} d x^{*} d x^{*},
\end{aligned}
$$

where $C_{1}=12 \frac{f_{2}\left(x^{*} h_{m}^{*}\right)}{f_{3}\left(x^{*} h_{m}^{*}\right)}-6 \frac{f_{1}\left(x^{*} h_{m}^{*}\right)}{f_{3}\left(x^{*} h_{m}^{*}\right)}$

$$
\begin{aligned}
\tau_{z x}^{*} & =\frac{\tau_{x} h_{0}}{\mu U} \\
& =\frac{\partial u^{*}}{\partial z^{*}}\left[1-l^{*} \frac{\partial^{2} u^{*}}{\partial z^{*^{2}}}\right] .
\end{aligned}
$$

The non dimensional shear force components acting upon the lower (Horizontal) and upper (vertical) surfaces are Figure (b) describe the dimensionless shear force components that is lower or horizontal as well as upper or vertical component as a function of fluid film thickness for different values of $l^{*}$.

It is shown that the shear force component along the lower surfaces decreases with increasing values of $h^{*}$, but the shear force component along both the surfaces increases with the fluid film thickness.

So the force vector component parallel to the cross section increases and it results with the loss of velocity. It increases with the fluid film thickness describes that there is a strong internal forces between the neighboring particles in the lower surface.

\section{Results and Discussions}

The Dimensionless couple stress parameter defined by $l^{*}=\frac{l}{h_{0}}=\frac{\left(\frac{\eta}{\mu}\right)^{2}}{h_{0}}$, where $\eta$ is the material constant responsible for the couple stress and $\mu$ is the classical viscosity coefficient has an effect on the steady-state performance of wideparabolic shaped slider bearings. The microstructure size is characterized by 


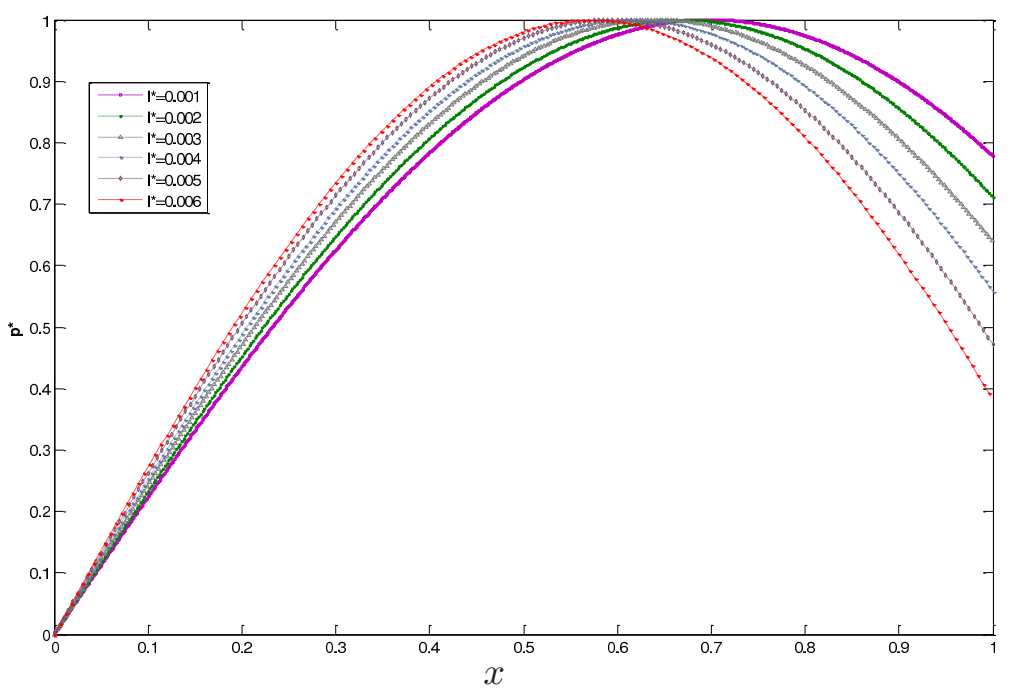

Figure 1: Variation of non-dimensional steady pressure distribution $p^{*}$ with couple stress parameter $l^{*}$.

the dimension of the ratio $\left(\frac{\eta}{\mu}\right)$. The couple stress parameter $l^{*}$ gives the interaction of the couple stress with the slider bearing geometry. As the value of $l^{*}$ approaches zero, the effects of couple stress vanish and it becomes the Newtonian lubricant case. In order to obtain the bearing characteristics, the numerical computations are performed by choosing the reference film thickness $h_{0}=h_{m}$, the slip parameter $s^{*}=0.01$ to $s^{*}=0.06$ and couple stress parameter $l^{*}=0.006$ to $l^{*}=0.006$.

Figure 1 shows the dimensionless film pressure $p^{*}$ as a function of $x^{*}$ for different values of $l^{*}$. It is observed that the effects of couple stresses at $l^{*}=$ 0.001 increase $p^{*}$ as compared when $l^{*}=0$. i.e. Newtonian case. The pressure distribution increases up to $x^{*}=0.6$ and reaches a stagnated point (saturation point) where the distribution is uniform and maximum. At this point the effects of couple stresses upon the film pressure are maximum. After that point the whole process getting reverse and it is maximum for $l^{*}=0.001$ and minimum for $l^{*}=0.001$.

This shows the basic characteristics of wide parabolic-shaped slider bearing over a traditional Newtonian lubricant case which shows improvement in the steady-state performance.

Figure 2: Steady State Performance: The variation of Non- Dimen- 


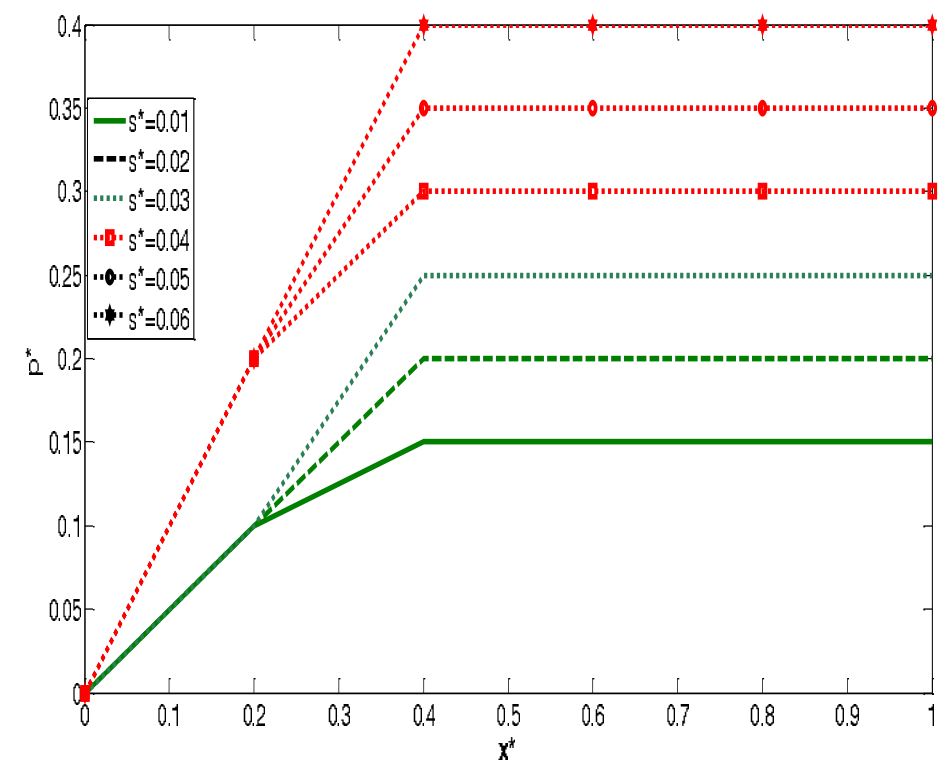

Figure 2: Variation of non-dimensional steady pressure distribution $p^{*}$ with slip parameter $s^{*}$.

sional steady-state pressure $p^{*}$ for different values of slip parameter. The effect of velocity slip is to decrease the resistance encountered by the lubricants flow in the fluid.

In Figure 2, the effect of slip parameter on a lubricant merges till $x^{*}=0.2$ and then shows a variation and then steady state performance.

It increases the pressure and improves the lubricant characteristic followed by increase in load capacity. The strongest slip decreases the resistance of lubricant flow with an extent that there is a lesser tendency for lubricant to flow through the porous material.

The effects of couple stresses upon the wide parabolic shaped slider bearings is of steady state performance and it shows the variations after certain point ( i.e., saturation point) which will help the engineers to design a bearing with multiple functions according to different characteristics. Further, this study makes it mandatory that the pressure distribution must be given due consideration while designing the bearing system even if a suitable value of slip parameter has been chosen.

Figure 3 shows, with the varying couple stress parameter, the distribution of load carrying capacity with respect to fluid film thickness. 


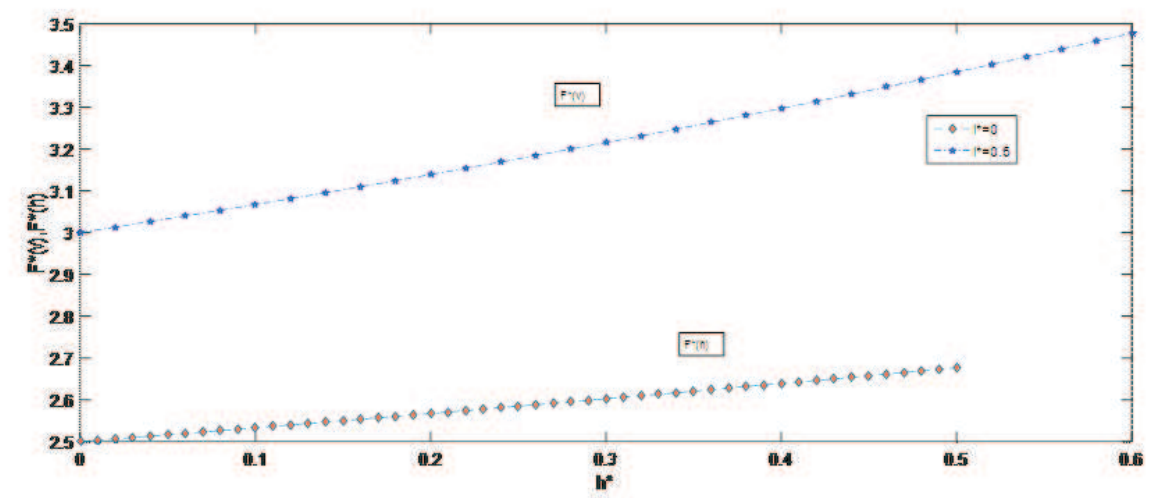

Figure 3: Variation of non-dimensional load carrying capacity with respect to couple stress parameter.

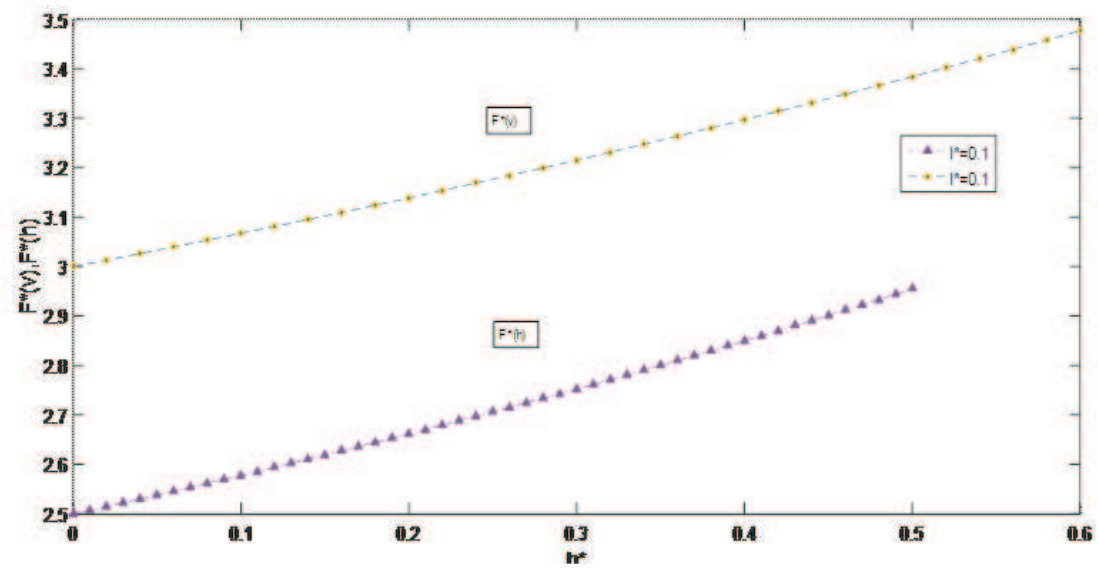

Figure 4: Variation of non-dimensional shear force components with respect to couple stress parameter.

The total energy output of a system increases with the increase in couple stress parameter as we consider the increase in thickness. It shows that at some point [or value of film thickness] the bearing shows no distribution, which states that the work load supported by a bearing can take the load to some extent and after that it doesn't show any characteristics.

Figure 4 shear force component with varying values of film thickness keeping 


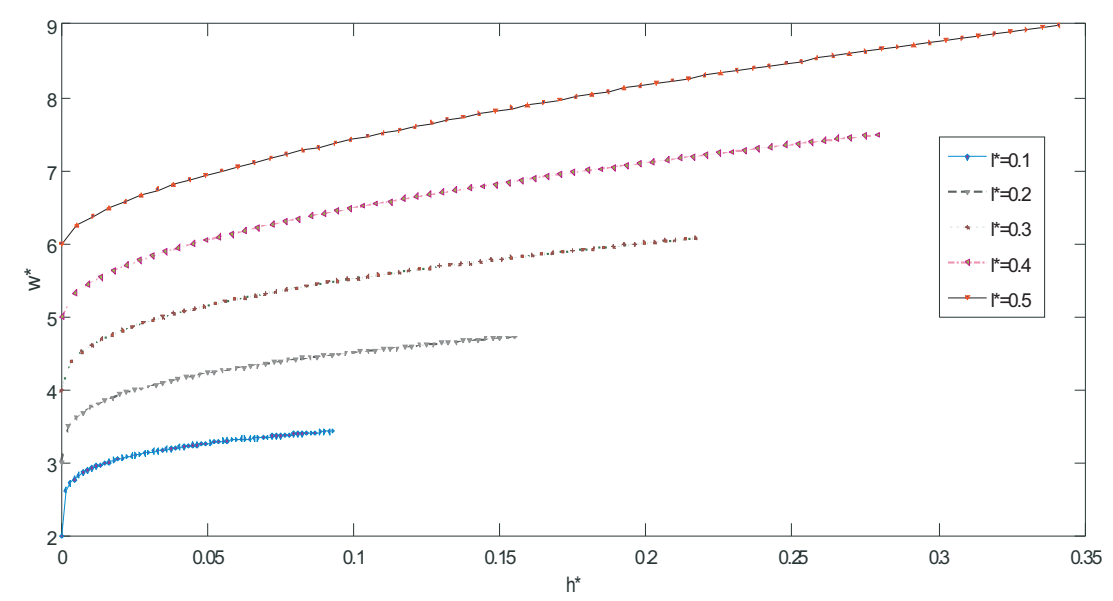

Figure 5: Variation of non-dimensional load carrying capacity with couple stress parameter.

couple stress parameter as constant.

Figure 5 shows the shear stress component along lower as well as upper surface with couple stress parameter values to be taken as minimum and maximum. As couple stress parameter value is $\operatorname{zero}(=0)$ i.e., a Newtonian case, the shear force along the boundary or lower surface (intermolecular forces between the neighboring particles) are less or in other words it is weak.

On the other side when we keep on increasing the value of couple stress parameter to the maximum i.e., $(=0.5)$ there is a strong intermolecular forces between the neighboring particles and the system (bearing), which leads to have a strong load carrying capacity of a bearing which shows the characteristics of bearings in any situation. So, we can conclude that couple stress as a lubricant act as a good lubricant and it enhances the life period of a bearing.

\section{Conclusion}

In this paper we studied the effect of the load carrying capacity and shear stress components (lower as well as upper surface) on the porous parabolic inclined slide bearing lubricated with couple stress parameter. The pressure of the plates with respect to distance showed a increase in pressure with increase in couple stress parameter which in turn leads to increase in the load carrying 
capacity which increases the life span of a bearing. Also we have considered the shear stress component which is important in understanding which part of the bearing is/are mostly stressed, it is basically helpful for the mechanics people to design the bearing so that there should be some balance equilibrium of forces on bearing. We found that when it is a Newtonian case, there is a sudden fall or drop of the forces between the particles with the bearing which shows that strain rate are related by a constant viscosity tensor that does not depend on the stress state and velocity of the flow.

We have seen the variation of distribution for pressure, the pressure increases with increase in slip parameter and reaches a stagnated state and shows a steady state with uniform behavior.

\section{Nomenclature}

$h$ - film thickness function,

$h_{m}-$ minimum film thickness at the outlet,

$k$ - permeability of the porous material,

$l$ - couple stress parameter,

$L$ - length of the bearing $p^{*}$ - pressure in the porous region,

$s$ - slip parameter,

$\eta$ - material constant responsible for the couple stress property,

$\mu$ - classical viscosity coefficient,

$U$ - sliding velocity of the lower part,

$x, y$ - Cartesian rectangular coordinate,

$u, v$ - velocity components,

$\tau_{z x}$ - shear stress on the plane,

$w$ - load carrying capacity,

$W$ - dimensionless load carrying capacity per unit width.

\section{References}

[1] P.S. Rao, S. Aggarwal, Effect of surface roughness on the hydrodynamic lubrication of porous inclined slider bearing considering slip velocity and squeeze velocity with couple stress fluids, International Journal of Engineering, science and technology, 6 (2014).

[2] Jaw-Ren Lin, Yu-Ming Lu, Steady state performance of wide parabolic shaped slider bearings with a couple stress fluids, Journal of Marine Science and Technology, 12 (2004).

[3] N.B. Naduvinamani, G.B. Marali, Numerical solution of couple stress full Reynolds equation for plane inclined porous slider bearings with squeezing effect, Canadian Journal of Pure and Applied Sciences, 1 (2007). 
[4] G. Ramanaiah, Squeeze films between finite plates lubricated by fluids with couple stress (1979), 315-320.

[5] H. Wu, An analysis of the squeeze film between porous rectangular plates, ASME Journal Lubrication Technology, 94 (1972), 64-68.

[6] G. Ramanaiah, A. Sundarammal, Effect of bearing deformation on the characteristics of a slider bearing, Wear, 78 (1982), 273-278.

[7] S.T. Tzeng, E. Saibel, Surface roughness effect on slider lubrication, ASLE Trans, 10 (1967), 334 .

[8] S. Sundarammal Kesavan, K.N. Santhana, Surface roughness effect on squeeze film behavior in porous transversely triangular plates with couple stress fluids, Int. J. Mech. Eng. Tech., 3, No. 2 (2012), 1-12.

[9] D.C. Das, Elastohydrodynamic lubrication theory of line contacts: Couple stress fluids, STLE Tribology, 24 (1997), 353-359.

[10] A. Cameron, The Principles of Lubrication, A. Longmans Green and Co., Ltd., London (1966).

[11] N.M. Bujurke, N.G. Naduvinamani, The lubrication of lightly cylinders in combined rolling, sliding and normal motion with couple stress fluid, Int. J. Mech. Sci., 32, 969979.

[12] V.K. Stokes, Couple stresses in fluids, Phys. Fluids, 9, 1709-1715.

[13] G. Ramanaiah, Slider bearings lubricated by fluids with couple stress, Wear, 52 (1979), $27-36$. 
